\title{
A Review on Prevalence of Medication Adherence among Hypertensive Patients in India
}

\author{
Satish S $\mathbf{S}^{1}$ Minnu Sara Sam², A R Shabaraya ${ }^{3}$ \\ ${ }^{1}$ Associate Professor, ${ }^{2}$ Student, PharmD, ${ }^{3}$ Professor \& Head, \\ Department of Pharmacy Practice, Srinivas College of Pharmacy, Mangalore, Karnataka-574143 \\ Corresponding Author: Minnu Sara Sam
}

\begin{abstract}
Prevalence of hypertension is increasing exponentially in India. Hence hypertension has become a crucial public health problem in India. High blood pressure (BP) could be a major risk factor for cardio vascular disease and better control can result in prevention of 300,000 of the 1.5 million annual deaths from cardiovascular diseases in India.

Various studies among Indian patients evident that not adherence to their antihypertensive regimen and this might end in poor blood pressure control. Adherence to medication among the hypertensive people from the current studies is poor. A comprehensive strategy to enhance adherence to antihypertensive medications is the need of the hour. In this article an attempt has been made to compile all the research evidence on prevalence of Medication Adherence among hypertensive patients in India.
\end{abstract}

Keywords: Medication, Adherence, Prevalence, Hypertension, India.

\section{INTRODUCTION}

WHO reports that adherence is the extent to which the patient follows medical instructions, takes medication, follows a prescribed diet and sticks to lifestyle changes which corresponds with the recommendations from healthcare provider. Non adherence in drug therapy can cause increased morbidity, mortality and an enormous cost to the health care system. India, is likely to face an enormous burden of Noncommunicable diseases (NCDs) in future; of these diseases, hypertension is one of the most important preventable and treatable causes of mortality and morbidity in the elderly population. Furthermore, high blood pressure (BP) is a modifiable risk factor for cardiovascular disease (CVD). ${ }^{[1]}$ Improving adherence has great impact on the population. The World Health Organization (WHO) describes poor adherence as the most vital cause of uncontrolled blood pressure and estimates that $50 \%$ of people don't take their antihypertensive medication as prescribed.

The prevalence of hypertension in India is estimated to be around 20\% - 40\% in urban and $12 \%-17 \%$ in rural areas. According to a World Health Organization (WHO) report, the prevalence of hypertension in India among men and women aged 25 years or more was $23.1 \%$ and $22.6 \%$ respectively. Hypertension is an asymptomatic condition, which result in delayed diagnosis and treatment especially among rural population. But better control can lead to prevention of 300,000 of the 1.5 million annual deaths from cardiovascular diseases in India. ${ }^{[2]}$

\section{Medication Adherence}

Accurate assessment of adherence behaviour is necessary for effective and efficient treatment planning, and for ensuring that changes in health outcomes are often attributed to the recommended regimen. Adherence is related to the way within which individuals judge personal need for a medication relative to their concerns about its potential adverse effect. ${ }^{[3]}$ 
Good adherence has been shown to enhance blood pressure control and reduce the complications of hypertension. Low patient compliance is one of the foremost important therapy-limiting factors in hypertension. ${ }^{[4]}$ Nonadherence can't only lead to adverse health outcomes but also increased healthcare costs. ${ }^{[5]}$ Non-adherence was an active decision, partly based on misunderstandings of the condition and general disapproval of medication, but mostly taken so as to facilitate daily life or minimize adverse effects. ${ }^{[6]}$ Inadequate access to medications or interruption of a person's pattern were related to the incidental missing of medications. ${ }^{[7]}$

Approximately $50 \%$ of patients don't take their medications as prescribed. Factors causative to poor medication adherence are myriad and include those that are related to patients (e.g., suboptimal health literacy and lack of involvement in the treatment decision-making process), those which are related to physicians (e.g., prescription of complex drug regimens, communication barriers, ineffective communication of information about adverse effects, and provision of care by multiple physicians), and those that are related to health care systems (e.g., office visit time limitations, limited access to care, and lack of health information technology). ${ }^{[8]}$ The WHO rates hypertension as one of the foremost important causes of premature death worldwide.

\section{Region Wise Prevalence of Medication Adherence}

The prevalence of hypertension has been exponentially increasing in India, whereas levels of awareness, treatment, and control remain low. ${ }^{[9]}$ Hence hypertension has become a significant public health problem in India. High blood pressure (BP) is a major risk factor for cardio vascular disease and better control can lead to prevention of 300,000 of the 1.5 million annual deaths from cardiovascular diseases in India. ${ }^{[2]}$

\section{Pune, Maharashtra}

The study by Nagarkar AM et.al., which was carried out in a region served by the state government's civil hospital located in the urban parts of Pune District in the state of Maharashtra used the Sample size of 209 diagnosed hypertensive patients was calculated using prevalence of $16.4 \%$. 4 incomplete records were deleted from final analysis hence present paper is based on 205 participants. For the purpose of further analysis lower adherence category was merged with medium and compared with high adherence category. Score 8 on scale was categorised as 'high adherence', those who scored below 8 as 'low adherent'. Thus, 23.4 percent were high adherent and 76.5 percent reported low adherence to the medication. In this study MMAS-8 is used with the intention to screen patients with different degree of medicine adherence. Studies from patient's perspective have brought out several reasons for poor adherence like prescribing complex regimens, failing to explain the benefits, side effects of a medication, cost of the medications and complexity of treatment in terms of number of medicines and number of times to administer in a day. ${ }^{[10]}$

\section{Ernakulam, Kerala}

A study by Bala Subhramanian A et.al. which was conducted among the rural population of Njarackal Panchayat, a coastal village in Ernakulam district of Southern India included 189 respondents. A total of 372 households were visited to attain the requisite sample size. High adherence was seen in $46 \%$ of the patients, while medium and low adherence was seen in $41.3 \%$ and $12.7 \%$, respectively. Risk factors of poor adherence identified were poor knowledge of the complications (OR-2.120; 95\% confidence interval [CI]1.110-4.048), availing government pharmacy (OR-2.379; 95\% CI1.131-5.004), and being asymptomatic at the time of diagnosis (OR$2.120 ; 95 \%$ CI 1.110-4.048). [11] In this study Morisky 4-item medication adherence questionnaire consists of a set of four 
questions with a yes or no answer was used to check the adherence.

\section{Moradabad, North India}

From the study by Siraj Ahmed et.al., which was carried out among adult hypertensive patients, attending the Urban Health and Training Centre (UHTC) for the assessment of factors responsible for adherence to antihypertensive treatment, out of 334 patients $195(58.4 \%)$ were male and $139(41.6 \%)$ were female. Good adherence to treatment was found more among males than among females $(\mathrm{OR}=1.25)$. Married patients had good adherence to antihypertensive treatment as compared to unmarried patients $(\mathrm{OR}=1.95)$. A positive family history of hypertension $(\mathrm{OR}=1.82)$, having awareness of hypertension complications $(\mathrm{OR}=4.15)$ and those having co-morbidities $(\mathrm{OR}=2.93)$ had good adherence. Good adherence to treatment was present in those patients who were either receiving one medicine per day $(\mathrm{OR}=4.27)$, or taking medicine once a day $(\mathrm{OR}=1.96)$. Poor adherence to antihypertensive treatment was seen in patients with unrestricted dietary habits $(\mathrm{OR}=0.33)$, salt intake of $>5 \mathrm{gm} /$ day $(\mathrm{OR}=0.47)$, and physical exercise less than 30 minutes per day $(\mathrm{OR}=0.59)$, the habit of alcohol consumption $(\mathrm{OR}=0.52)$, tobacco chewing $(\mathrm{OR}=0.73)$, and smoking $(\mathrm{OR}=0.40)$. Good adherence to treatment was present in those patients who were either receiving one medicine per day $(\mathrm{OR}=4.27)$, or taking medicine once a day $(\mathrm{OR}=1.96) .{ }^{[12]}$ The Morisky 8-Item Medication Adherence Questionnaire was used to assess the level of adherence to the antihypertensive medication.

\section{Mangalore, Karnataka}

A study by Nithin kumar et.al.,
which was conducted among the hypertensive patients attending the peripheral outreach clinics belonging to the Department of Community Medicine, and the Medicine OPD at the hospital attached to Kasturba Medical College, Mangalore included total of 120 hypertensive patients.
They were assessed about their level of adherence towards antihypertensive medication giving a response rate of $100 \%$. on assessing the adherence level using Morisky scale it was observed that $54.2 \%$ of the participants had a medium level of adherence and $45.8 \%$ had poor level of adherence to their medication. There were no participants with high level of adherence to antihypertensive medication. ${ }^{[13]}$

\section{West Bengal}

The study by sahoo et.al., was a descriptive, cross-sectional and clinic based and it was conducted in two health centres in Singur block, West Bengal. It included total of 186 study subjects majority i.e. $46.8 \%$ belonged to $60-64$ years age group, followed by $22.6 \%$ in $65-69$ years age group and mean age \pm SD of the population was $66.88 \pm 6.65$ years. $44.63 \%$ of the respondents adhered to anti-hypertensive drugs. The schedule used for data collection had two parts: The first part consists of Background information of the study population (i.e Demographic and socioeconomic profile, co-morbidities, treatment history and the causes for poor adherence. The second part consist of Morisky Medication Adherence Scale (MMAS-8) which is a standardised scale to measure the drug adherence. As per MMAS score 55.4\% were highly adherent (score=8) and $32.3 \%$ and $12.3 \%$ belong to the category of medium $(6$ to $<8)$ and low adherence $(<6)$ respectively. The most common reasons for poor adherence were due to irregular supply from government pharmacy (72.3\%) followed by forgetfulness $(67.5 \%)$ and nonaffordability $(66.3 \%)$. Poor adherence to drugs was significantly $(\mathrm{p}<0.05)$ associated with contextual variables like age, literacy, socio-economic status, duration of treatment and presence of comorbidities. ${ }^{[14]}$

\section{Hyderabad, Telangana}

A cross-sectional study by DR. Babu rao was conducted in Urban Slum area of Hyderabad included total of 220 individuals aged 20 years and above on antihypertensive treatment for more than 6 
months were interviewed. Better adherence and a statically significant association were found in patient above 60 years of age $(67.2 \%)$, married people $(84.7 \%)$, nonsmokers $(74.8 \%)$ and non-tobacco users (68.9\%) and among people consuming 1 tablet a day $(67.7 \%)$. The data was collected using a pretested structured questionnaire consisting of 3 sections: Section IParticipants general information, Section IIDiagnosis and treatment details of hypertension, and Section III -The 4-item Morisky questionnaire. ${ }^{[15]}$

\section{Guntur, Andhra Pradesh}

A institutional based cross sectional study by Hema K, et.al., included 400 hypertensive patients using systematic random sampling. The overall adherence of study population among study population was $15.3 \%(n=61)$. Adherence was found to be higher in 50 years \& above age group (46.6\%) and among females (51.6\%). Similarly, adherence was more among those who belonged to nuclear families $(47.1 \%)$ and among literates (44.2\%). adherence to anti-hypertensive medication which was measured using an eight item Morisky Medication Adherence Scale (MMAS). ${ }^{[16]}$

\section{Kancheepuram, Tamilnadu}

A community-based cross-sectional study by J. Venkatachalam et.al., was conducted in a rural area of Kancheepuram district, Tamil Nadu included 473 hypertensive patients of which 226 were males and 247 were females. The prevalence of adherence was $24.1 \%$ ( $n=$ 114) within the study population. Respondents with routine physical activity, non-smokers and non-alcoholics were more adherent to HT medication as compared with respondents with sedentary lifestyle, smoking and alcohol intake $(\mathrm{P}<0.005)$. Based on health belief model, the respondents who perceived high susceptibility, severity, benefit had better adherence compared with moderate and low susceptibility, severity, benefit. ${ }^{[17]}$

\section{DISCUSSION}

Hypertension imposes severe financial and service burdens on health systems. As per a systematic review, the pooled percentage of adherence among hypertensives of low- and middle-income countries is $36.65 \%$, while in a high-income country like the United States, it is $51 \%$. Adequate control of blood pressure is a key element in the prevention of hypertension induced-organ damage and life-threatening complications. Effective treatment can considerably minimize the incidence of subsequent adverse clinical outcomes. Nonadherence to antihypertensive medication is one of the main reasons for failure to control blood pressure among those on treatment and is usually associated with bad outcomes of the disease. The matter of non-adherence is universal and not just limited to developed or developing countries. Empowering the patients with correct knowledge about the disease, its treatment and benefits can help to overcome the misguided cultural beliefs and associated misconceptions.

\section{CONCLUSION}

From the review it can be concluded that various studies among Indian patients evident that not adherence to their antihypertensive regimen and this might result in poor blood pressure control. Adherence to medication among people with hypertension from the current studies is poor. Apart from subsidising medications, innovative health education modality has to be developed to attain the better outcome.

\section{Acknowledgement: None}

\section{Conflict of Interest: None}

Source of Funding: None

Ethical Approval: Not Applicable

\section{REFERENCES}

1. Fuchs FD, Whelton PK. High Blood Pressure and Cardiovascular Disease. Hypertension. 2020;75(2):285-292. 
2. Gupta R, Gupta VP. Hypertension epidemiology in India: lessons from Jaipur Heart Watch studies. Curr Science. 2009; 97: $349-55$

3. Adherence to long-term therapies: evidence for action. Geneva: WHO; 2003.

4. Lüscher TF, Vetter H, Siegenthaler W et al., Compliance in hypertension: facts and concepts. J Hypertens Suppl. 1985;3(1):S39.

5. Pasma A, Schenk C, Timman R et al., Does non-adherence to DMARDs influence hospital-related healthcare costs for early arthritis in the first year of treatment? PLoS One. 2017;12(2)

6. Staffan Svensson, Karin I Kjellgren, Johan Ahlner et al., Reasons for adherence with antihypertensive medication International Journal of Cardiology. 2000;76(2-3): 157163.

7. Spencer, Karen. (2004). Assessment, Objectivity, and Interaction: The Case of Patient Compliance with Medical Treatment Regimens. Social Psychology Quarterly. 2004; 67(4):343-368.

8. Marie $\mathrm{T}$, Brown $\mathrm{MD}$, Jennifer $\mathrm{K}$ et al., Medication Adherence: WHO Cares? Mayo Clinic Proceedings. 2011;86(4): 304-314.

9. Kharde AL, Deshpande J, Phulambrikar R et al., Prevalence of hypertension and its risk factors in a field practice area of tertiary care teaching hospital in rural area of Western Maharashtra. Int J Med Sci Public Health 2018;7(2):76-79.

10. Nagarkar AM, Gadhave SA, Sharma I et al., Factors Influencing Medication Adherence among Hypertensive Patients in a Tertiary Care Hospital, Pune, Maharashtra. Natl J Community Med. 2013; 4(4): 559-63.

11. Balasubramanian A, Nair SS, Rakesh PS et al., Adherence to treatment among hypertensives of rural Kerala, India. J Family Med Prim Care. 2018;7:64-9

12. Siraj Ahmad. Assessment of adherence to antihypertensive treatment among patients attending a health care facility in North India Int J Res Med. 2015; 4(1):117-124

13. Nithin Kumar, Bhaskaran Unnikrishnan, Rekha Thapar et al., Factors associated with adherence to antihypertensive treatment among patients attending a tertiary care hospital in Mangalore, south India. 2014; 06(10): 2-14

14. Sahoo SK, Preeti PS, Biswas D et al., Adherence to Anti-Hypertensive Drugs: A
Clinic Based Study among Geriatric Hypertensive Patients in Rural, India. Natl J Community Med. 2018; 9(4): 250-254

15. Babu Rao, Pratyush R Kabra, Sreedhar M et al., Factors associated with adherence to antihypertensive treatment among hypertensive persons in a urban slum area of Hyderabad Indi. Indian journal of basic and applied medical research. 2014; 4(1): 471477

16. Hema K, Padmalatha P. Adherence to medication among Hypertensive patients attending a tertiary care hospital in Guntur, Andhra Pradesh. Indian Journal of Basic and Applied Medical Research. 2014; 4(1): 451- 456

17. Venkatachalam J, Abrahm SB, Singh $Z$ et al., Determinants of Patient's Adherence to Hypertension Medications in a Rural Population of Kancheepuram District in Tamil Nadu, South India. Indian Journal of Community Medicine: Official Publication of Indian Association of Preventive \& Social Medicine. 2015;40(1):33-37

18. Assessment of burden of non-communicable diseases in India. Final report of Project New Delhi: Indian Council of Medical Research; 2004.WHO India.

19. Horne R, Weinman J. Patients' beliefs about prescribed medicines and their role in adherence to treatment in chronic physical illness. Journal of Psychosomatic Research. 1999;47:555-567

20. Psaty BM, Koepsell TD, Wagner EH et al., The relative risk of incident coronary heart disease associated with recently stopping the use of beta-blockers. JAMA. 1990; 263 (12):1653-1657.

21. Rogers PG, Bullman W. Prescription medicine compliance: review of the baseline of knowledge-report of the National Council on Patient Information and Education. Journal of Pharmacoepidemiology. 1995; $3: 3-36$.

22. Demyttenaere K, Haddad P. Compliance with antidepressant therapy and antidepressant discontinuation symptoms. Acta Psychiatrica Scandinavica. 2000; 403:50-56.

23. Kaplan EM. Antidepressant noncompliance as a factor in the discontinuation syndrome. Journal of Clinical Psychiatry.1997; 58:3135.

24. Mansi Patil, Manuel Durairaj. Risk factors of hypertension among adult men: Evidence 
from a real-world outcomes investigation in a Western Indian population. International Journal of Advanced Research.2015; 3(7): 274-282

25. Victor RG, Leonard D, Hess P et al., Factors associated with hypertension awareness, treatment, and control in Dallas County, Texas. Arch Intern Med. 2008; 168 (12): 1285-9

26. Mary Jayne Johnson, Mary Williams, Elaine S. Marshall. Adherent and Nonadherent
Medication-Taking in Elderly Hypertensive Patients Clinical Nursing Research. 1999;8(4)

How to cite this article: Satish S, Sam MS, A R Shabaraya. A review on prevalence of medication adherence among hypertensive patients in India. International Journal of Research and Review. 2021; 8(7): 30-35. DOI: https://doi.org/10.52403/ijrr.20210706 